\title{
PRODUÇÃO DE MATERIAL DE REFERÊNCIA CERTIFICADO DE ÓXIDO DE HÓLMIO(III) EM AMPOLAS
}

\author{
Debora E. F. Gonçalves ${ }^{\mathrm{a}}$, Ana P. D. Alvarenga ${ }^{\mathrm{b}}$, Paulo P. Borges ${ }^{\mathrm{c}, *}$, Juliana F. S. Gomes ${ }^{\mathrm{b}}$ e Thiago O. Araujo ${ }^{\mathrm{c}}$ \\ ${ }^{a}$ Departamento de Material de Referência, Visomes Comercial Metrológica, 04823-080 São Paulo - SP, Brasil \\ ${ }^{b}$ Divisão de Metrologia Óptica, Instituto Nacional de Metrologia, Qualidade e Tecnologia, 25250-020 Duque de Caxias - RJ, Brasil \\ 'Divisão de Metrologia Química e Térmica, Instituto Nacional de Metrologia, Qualidade e Tecnologia, 25250-020 Duque de \\ Caxias - RJ, Brasil
}

Recebido em 11/06/2018; aceito em 30/08/2018; publicado na web em 24/09/2018

\begin{abstract}
PRODUCTION OF CERTIFIED REFERENCE MATERIAL OF HOLMIUM(III) OXIDE IN AMPOULES. This work reports the development of a certified reference material (CRM) of holmium(III) oxide $\mathrm{Ho}_{2} \mathrm{O}_{3}$ in acidic solution for the wavelength scale calibration of UV-Vis spectrophotometers. A $4.0 \% \mathrm{Ho}_{2} \mathrm{O}_{3}$ solution was gravimetrically prepared in $10 \%$ perchloric acid and bottled in $5.0 \mathrm{~mL}$ glass ampoules. Homogeneity, stability and characterization were assessed according to ISO 17034 and ISO Guide 35 . The material certification was undertaken by two laboratories by using UV-Vis spectrophotometry for characterization of the 14 peaks of minimum transmittance of $\mathrm{Ho}_{2} \mathrm{O}_{3}$, using a spectral bandwidth of $1.00 \mathrm{~nm}$ and a scanning speed of $30.0 \mathrm{~nm} \mathrm{~min}^{-1}$. The material property values in wavelength $(\mathrm{nm})$ were obtained for these 14 peaks, together with the expanded uncertainty $(U)$ of $0.12 \mathrm{~nm}$, for a coverage factor $(k=2.00)$ corresponding to a probability of approximately $95 \%$, which took into account the standard uncertainties obtained in each study performed for the certification of the produced reference material.
\end{abstract}

Keywords: holmium(III) oxide; certified reference material; spectrophotometry; wavelength; calibration.

\section{INTRODUÇÃO}

Os materiais de referência certificados (MRCs) são ferramentas importantes para o sistema de qualidade de um laboratório na garantia da confiabilidade de resultados e da exatidão de medições químicas. Possuem uma declaração de sua incerteza associada e a rastreabilidade metrológica demonstrável a padrões nacionais ou internacionais. ${ }^{1}$ A produção de um MRC envolve muitas etapas, pois ele deve ser planejado, desenvolvido, ter a homogeneidade e a estabilidade avaliadas, além de seu valor de propriedade determinado na etapa de caracterização e ter sua incerteza estabelecida. Dentre as diversas áreas que utilizam MRCs, destaca-se a área de óptica com a espectrofotometria no UV-Vis. Essa técnica é muito utilizada e o espectrofotômetro é um equipamento fundamental para a análise de diversos materiais. Por meio da espectrofotometria, compostos químicos podem ser identificados e quantificados por seus espectros característicos na faixa do ultravioleta (UV) ao visível (Vis). Como as absorbâncias associadas a diversos analitos podem ser alteradas em intervalos pequenos de comprimentos de onda, e a precisão e exatidão das medidas devem ser maximizadas, a calibração do espectrofotômetro é essencial. Para isto, são utilizados MRCs específicos para calibrar a escala de comprimentos de onda do equipamento como, por exemplo, a solução de óxido de hólmio(III), $\mathrm{Ho}_{2} \mathrm{O}_{3}{ }^{2}$

Apesar de não haver produção de MRCs no Brasil para a escala de comprimentos de onda, existem outros MRCs que podem ser utilizados na calibração e checagem da escala de comprimentos de onda de espectrofotômetros UV-Vis, como o filtro sólido de hólmio e de didímio. ${ }^{3}$ Atualmente, o MRC de $\mathrm{Ho}_{2} \mathrm{O}_{3}$ em solução é o material mais utilizado, já que ele pode ser utilizado na faixa do UV-Vis e seus picos de absorbância são intrínsecos e bem resolvidos, ao contrário do filtro sólido de didímio. ${ }^{3} \mathrm{O}$ MRC de $\mathrm{Ho}_{2} \mathrm{O}_{3}$ em solução provê linhas mais nítidas e menos dependentes de matriz do que o filtro sólido de hólmio. ${ }^{4}$ Ainda, algumas normas e farmacopeias exigem o uso da solução de $\mathrm{Ho}_{2} \mathrm{O}_{3}$ para a verificação da escala de comprimento de onda. ${ }^{5,6}$

\footnotetext{
*e-mail: ppborges@inmetro.gov.br
}

Este trabalho tem como objetivo apresentar os resultados da produção do MRC de solução de $\mathrm{Ho}_{2} \mathrm{O}_{3}$ em ampolas de vidro, primordial para garantir a rastreabilidade e confiabilidade dos resultados das medições espectrofotométricas.

\section{PARTE EXPERIMENTAL}

\section{Materiais}

Para a produção e estudo do candidato a MRC, foram empregados o óxido de hólmio $\left(\mathrm{Ho}_{2} \mathrm{O}_{3}\right)$ com pureza de 99,99\% (Alfa Aesar, EUA), o ácido perclórico $\left(\mathrm{HClO}_{4}\right)$ P.A. (Merck, Alemanha) e a água desionizada, com condutividade menor ou igual a $1,00 \mu \mathrm{S} \mathrm{cm}{ }^{-1}$, obtida com purificador de água (Elga, Inglaterra). $\mathrm{O} \mathrm{Ho}_{2} \mathrm{O}_{3}$ de pureza $99,99 \%$ foi escolhido, pois estudos preliminares ${ }^{7}$ indicaram que não houve diferenças espectrais entre $\mathrm{Ho}_{2} \mathrm{O}_{3}$ de pureza $99,99 \%$ e $99,999 \%$ na região espectral estudada. Foram utilizados uma balança analítica digital com resolução de $0,1 \mathrm{mg}$ (Mettler Toledo, EUA), banho termostático (Lauda, Alemanha), máquina de selagem de ampola (Rota Verpackungstechnik, Alemanha), espectrofotômetro digital UV-Vis, modelo PV-667-0 (Agilent, EUA), espectrofotômetro digital UV-Vis-NIR, modelo PR-092 (Perkin Elmer, EUA) e MRC de $\mathrm{Ho}_{2} \mathrm{O}_{3}-\mathrm{SRM}-2034$ (NIST, EUA).

\section{Etapas da certificação do $\mathrm{MRC}$ de $\mathrm{Ho}_{2} \mathrm{O}_{3}$}

As etapas de processamento do material, avaliação da homogeneidade, avaliação da estabilidade, caracterização, avaliação da incerteza e atribuição dos valores de propriedade e suas incertezas são discutidas a seguir.

\section{Preparo da solução de $\mathrm{Ho}_{2} \mathrm{O}_{3}$}

$\mathrm{O}$ procedimento de produção da solução de $\mathrm{Ho}_{2} \mathrm{O}_{3}$ foi o adotado pelo NIST, conforme Weidner et al. ${ }^{7,8}$ A concentração de solução de $\mathrm{Ho}_{2} \mathrm{O}_{3}$ preparada foi de $4,0 \%(\mathrm{~m} / \mathrm{m})$ em $10 \%(\mathrm{~m} / \mathrm{m})$ 
$\mathrm{HClO}_{4}$. Inicialmente, $\mathrm{Ho}_{2} \mathrm{O}_{3}$ foi pesado em balança analítica digital diretamente em um Erlenmeyer. Aproximadamente 1/10 da água desionizada a ser utilizada foi adicionada ao Erlenmeyer contendo o $\mathrm{Ho}_{2} \mathrm{O}_{3}$. A quantidade total de $\mathrm{HClO}_{4}$ foi pesada em um recipiente de vidro e adicionada ao Erlenmeyer. A mistura foi colocada em banho termostático à temperatura de $80^{\circ} \mathrm{C}$, durante $1 \mathrm{~h}$. Após a obtenção de uma solução homogênea e transparente, o Erlenmeyer foi retirado do aquecimento e deixado resfriar à temperatura ambiente.

A solução de $\mathrm{Ho}_{2} \mathrm{O}_{3}$ foi transferida quantitativamente para um balão de vidro, e água desionizada foi adicionada até a indicação da massa total de solução desejada. A solução foi homogeneizada e mantida a $(20 \pm 5){ }^{\circ} \mathrm{C}$ até o envase do material.

A solução de $\mathrm{Ho}_{2} \mathrm{O}_{3}$ preparada foi envasada em ampolas de vidro transparente de 5,0 $\mathrm{mL}$. A primeira etapa do envase foi a abertura das ampolas com ajuda de uma chama da máquina de selagem. O enchimento das ampolas com a solução de $\mathrm{Ho}_{2} \mathrm{O}_{3}$ foi realizado utilizando um repipetador automático. Antes de fechá-las, a máquina de selagem injetou argônio na ampola, para evitar reações com o oxigênio do ar. A seguir, as ampolas com a solução foram colocadas no carrossel da máquina de selagem para fechamento da sua parte superior. As ampolas foram identificadas com numeração de acordo com a ordem de envase, totalizando 60 unidades e armazenadas a $(25 \pm 5){ }^{\circ} \mathrm{C}$.

\section{Estudos de certificação do material de referência de $\mathrm{Ho}_{2} \mathrm{O}_{3}$}

Para a certificação do lote de solução de $\mathrm{Ho}_{2} \mathrm{O}_{3}$ produzida, a espectrofotometria no UV-Vis foi utilizada na avaliação da homogeneidade e da estabilidade e na caracterização, sendo analisados os 14 picos de máxima absorbância da solução de $\mathrm{Ho}_{2} \mathrm{O}_{3}$. Os parâmetros de medição utilizados foram: modo: Scan, faixa espectral: $235 \mathrm{~nm}$ a $645 \mathrm{~nm}$, largura de banda espectral: $1,00 \mathrm{~nm}$ e velocidade de varredura: $30,0 \mathrm{~nm} \mathrm{~min}{ }^{-1}$. Para cada ampola analisada, foram realizados 2 ciclos de medição, 3 repetições para cada ciclo, num total de 6 medições.

\section{Avaliação da homogeneidade}

Para a avaliação da homogeneidade, utilizou-se o espectrofotômetro PV-667-0. Todas as ampolas foram medidas no mesmo dia para garantir melhor repetibilidade.

A ferramenta estatística de regressão linear foi empregada para verificar se houve tendência na seleção das unidades. ${ }^{9} \mathrm{O}$ teste estatístico ANOVA foi utilizado para avaliar a homogeneidade do lote produzido, levando em conta a variabilidade dos resultados obtidos tanto entre as ampolas quanto dentro das ampolas. ${ }^{10} \mathrm{Com}$ a tabela ANOVA, foram obtidos os valores de $M Q$ (média quadrática) entre e dentro das unidades, $g l$ (graus de liberdade) entre e dentro das unidades, $F_{\text {calculado }}$ e $p$-valor, necessários para avaliar a homogeneidade e calcular a incerteza referente a esse estudo.

\section{Avaliação da estabilidade}

Após a comprovação da homogeneidade do lote de soluções de $\mathrm{Ho}_{2} \mathrm{O}_{3}$, foi realizada a avaliação da estabilidade do lote, a curto e a longo prazos, utilizando o espectrofotômetro PV-667-0. Para análise dos dados, a ferramenta estatística de regressão linear ${ }^{9}$ foi empregada para verificar a tendência dos resultados de comprimento de onda com o passar do tempo.

A estabilidade a curto prazo é associada a qualquer efeito devido ao transporte do material, na qual simula-se condições às quais o material possa vir a ser exposto durante o transporte. Os estudos de estabilidade a curto prazo foram realizados de modo isócrono (garantindo melhor repetibilidade), com duração de 35 dias (5 semanas), nas temperaturas $50{ }^{\circ} \mathrm{C}, 25^{\circ} \mathrm{C}$ e $4{ }^{\circ} \mathrm{C}$.
A estabilidade a longo prazo é associada a qualquer efeito devido ao armazenamento do material, na qual simula-se condições de prateleira. Os estudos de estabilidade a longo prazo foram realizados pelo modo clássico (garantindo uma precisão intermediária), com duração de 9 meses consecutivos.

\section{Caracterização}

Após a avaliação da estabilidade a curto prazo, foi realizada a caracterização do candidato a MRC produzido. Os resultados foram obtidos através da caracterização do mensurando utilizando 2 métodos independentes em 2 laboratórios, ${ }^{10}$ através do ensaio realizado pelo Laboratório de Radiometria e Fotometria (Laraf) do Inmetro (com utilização do espectrofotômetro PR-092), como também por medidas realizadas pelo Laboratório de Óptica da Visomes (com utilização do espectrofotômetro PV-667-0).

Os métodos utilizados para se determinar os comprimentos de onda do $\mathrm{Ho}_{2} \mathrm{O}_{3}$ foram independentes, e com a declaração da incerteza-padrão associada pelos 2 laboratórios. A compatibilidade dos resultados dos laboratórios foi verificada através de análise pelo índice zeta. ${ }^{11}$ Após os resultados serem comprovadamente compatíveis, os valores de propriedade do comprimento de onda dos picos de máxima absorbância da solução de $\mathrm{Ho}_{2} \mathrm{O}_{3}$ produzida foram atribuídos por meio de média ponderada, ${ }^{12}$ considerando os valores obtidos pelos laboratórios e suas incertezas padrão associadas à etapa de caracterização.

\section{Avaliação da incerteza}

Para avaliar a incerteza expandida $\left(U_{\text {MRC }}\right)$ da solução de $\mathrm{Ho}_{2} \mathrm{O}_{3}$ produzida, levaram-se em consideração os resultados obtidos em cada um dos estudos de certificação do material: homogeneidade, estabilidade e caracterização. As incertezas padrão referentes à homogeneidade $\left(u_{\text {hom }}\right)$, à estabilidade a curto prazo $\left(u_{\mathrm{ec}}\right)$ e à estabilidade a longo prazo $\left(u_{\mathrm{el}}\right)$ foram calculadas conforme ISO Guide $35,{ }^{13}$ e aquela referente à caracterização $\left(u_{\text {car }}\right)$ foi calculada por ponderação das incertezas obtidas de cada laboratório. Levando em consideração as fontes citadas, a incerteza expandida do MRC produzido foi calculada conforme Equação (1).

$$
U_{\mathrm{MRC}}=k \times \sqrt{u_{\mathrm{hom}}^{2}+u_{\mathrm{ec}}^{2}+u_{\mathrm{el}}^{2}+u_{\mathrm{car}}^{2}}
$$

em que $U_{\text {MRC }}$ é a incerteza expandida do MRC e $k$ é o fator de abrangência (de valor igual a 2,00 para $95 \%$ de probabilidade de abrangência).

\section{RESULTADOS E DISCUSSÃO}

O espectro de absorbância da solução de $\mathrm{Ho}_{2} \mathrm{O}_{3}$ preparada (Figura 1) inclui os 14 picos de máxima absorbância utilizados para calibração de espectrofotômetros na faixa espectral de $240 \mathrm{~nm}$ a $640 \mathrm{~nm}$ identificados por números.

\section{Homogeneidade do candidato a MRC}

Com as 10 ampolas selecionadas para a avaliação da homogeneidade, verificou-se que não houve tendência na seleção das unidades utilizando a regressão linear. A análise da homogeneidade dentro e entre as unidades se baseou na ANOVA, obtendo-se os valores de $F_{\text {calculado }}$ e $p$-valor. Para $\alpha=0,05, g l_{\text {entre }}=9$ e $g l_{\text {dentro }}=50$, o $F_{\text {crítico }}$ é de 2,12 . Para que o lote seja considerado homogêneo, o $F_{\text {crítico }}$ deve ser maior que $F_{\text {calculado }}$, e o $p$-valor deve ser maior que 0,05 (probabilidade de $95 \%$ de abrangência). Os dados de $F_{\text {calculado }}$ e $p$-valor para os 14 picos da solução de $\mathrm{Ho}_{2} \mathrm{O}_{3}$ produzida encontram-se na Tabela 1 . 


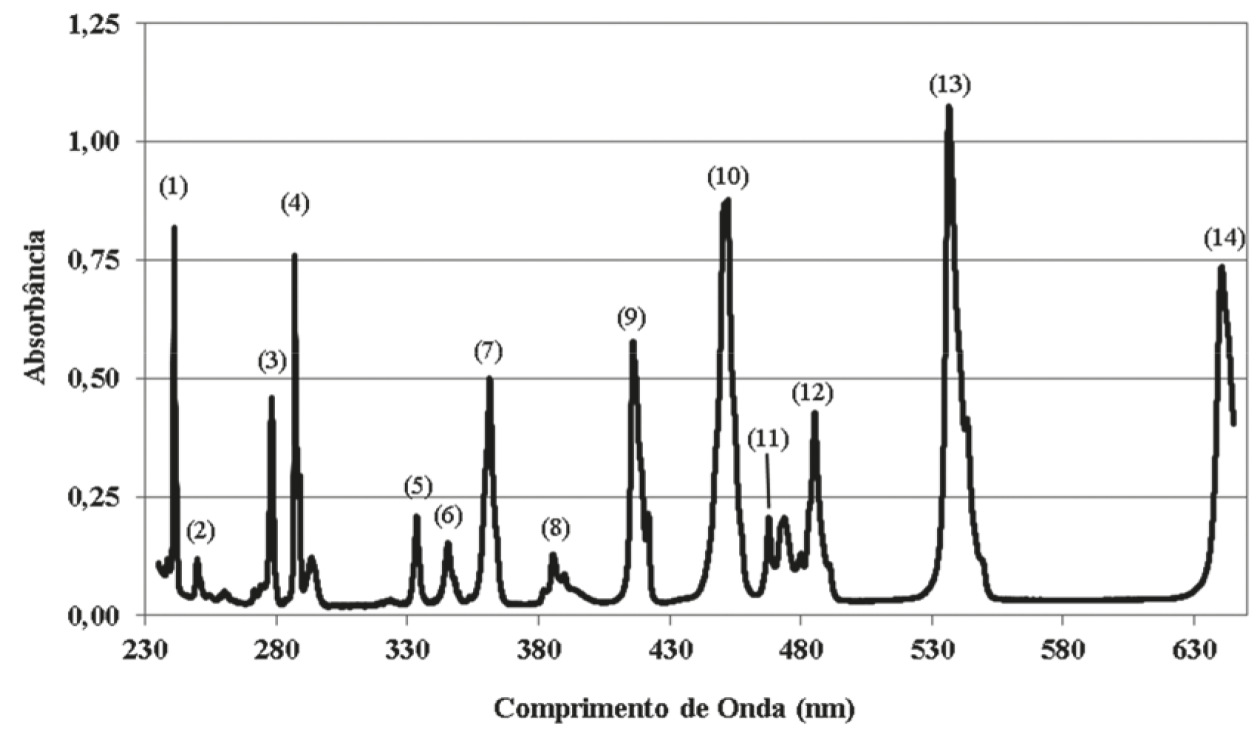

Figura 1. Espectro de absorbância da solução de $\mathrm{Ho}_{2} \mathrm{O}_{3}$ e os 14 picos de máxima absorbância usualmente certificados

Tabela 1. Valores para o teste F e p-valor referentes à avaliação da homogeneidade do lote candidato a MRC, para os 14 picos especificados na Figura 1

\begin{tabular}{ccc}
\hline $\begin{array}{c}\text { Valor nominal } \\
\text { do pico }(\mathrm{nm})\end{array}$ & $F_{\text {calculado }}$ & p-valor \\
\hline 241 & 1,87 & 0,08 \\
249 & 1,93 & 0,07 \\
278 & 1,62 & 0,13 \\
287 & 0,81 & 0,61 \\
333 & 0,75 & 0,66 \\
345 & 0,96 & 0,48 \\
361 & 0,65 & 0,75 \\
385 & 0,91 & 0,52 \\
416 & 1,89 & 0,08 \\
451 & 1,37 & 0,23 \\
467 & 1,40 & 0,21 \\
485 & 1,11 & 0,38 \\
536 & 0,27 & 0,98 \\
640 & 0,93 & 0,51 \\
\hline
\end{tabular}

Como $F_{\text {crítico }}>F_{\text {calculado }}$ e $p$-valor $>0,05$ para os 14 picos analisados, o lote da solução de $\mathrm{Ho}_{2} \mathrm{O}_{3}$ foi considerado homogêneo.

\section{Estabilidade a curto prazo do candidato a MRC}

Para o estudo de estabilidade a curto prazo, inicialmente, os resultados foram analisados pela técnica estatística de regressão linear, na qual obteve-se o valor do coeficiente angular $\left(\beta_{l}\right)$ e do erro padrão $\left(s \beta_{l}\right)$. Para indicar estabilidade, o valor de $\beta_{I}$ (em módulo) deve ser menor que o produto entre o $s \beta_{1}$ e o valor de $t_{\mathrm{st}}$ ( $t$-student, considerando aproximadamente $95 \%$ de confiança).

Na Tabela 2, são apresentados os valores de $\left|\beta_{l}\right|$ e $s \beta_{1} t_{\mathrm{st}}$. Verificase que, para 8 picos de máxima absorbância da solução de $\mathrm{Ho}_{2} \mathrm{O}_{3}$ (assinalados com a letra $a$ na Tabela 2), a condição $\left|\beta_{l}\right|<s \beta_{l} t_{\mathrm{st}}$ não foi atendida.

Com isso, foi necessário verificar se a tendência apresentada nesse estudo foi tecnicamente significativa, utilizando a Equação (2). Estipulou-se que a diferença entre $Y_{\text {estimado }}$ e $Y_{0}$ deveria ficar menor que
$30 \%$ da incerteza alvo de $0,15 \mathrm{~nm}$ para que a tendência encontrada no estudo não fosse considerada significativa.

$$
\% \text { dif }=\frac{\left|Y_{\text {estimado }}-Y_{0}\right|}{0,15} \times 100
$$

em que $Y_{\text {estimado }}$ equivale ao comprimento de onda obtido pela regressão linear estimando um tempo de 5 semanas (duração do estudo) e $Y_{0}$ é valor médio de comprimento de onda obtido nas leituras da primeira ampola. As diferenças relativas obtidas resultaram em valores menores que $30 \%$, indicando que a inclinação da reta não gerou instabilidade suficiente para que o valor de comprimento de onda saísse da faixa de incerteza da solução. Ou seja, para um prazo de 35 dias esses picos foram considerados estáveis. Por meio desse estudo, as temperaturas máxima e mínima de transporte do material puderam ser definidas como $50{ }^{\circ} \mathrm{C}$ e $4{ }^{\circ} \mathrm{C}$, respectivamente, sendo que o material pode ficar exposto às temperaturas limites por até 5 semanas, o tempo total de estudo.

\section{Estabilidade a longo prazo do candidato a MRC}

A avaliação da estabilidade a longo prazo foi realizada durante 9 meses consecutivos. Os dados passaram por regressão linear, na qual obtiveram-se os valores de $\beta_{l}$ e $s \beta_{l}$. Para indicar estabilidade, o $\beta_{1}$ (em módulo) deve ser menor que o produto $s \beta_{1} t_{\mathrm{st}}$. Na Tabela 3, encontram-se os valores de $\left|\beta_{l}\right|$ e $s \beta_{1} t_{\mathrm{st}}$

Para o estudo de estabilidade a longo prazo, verifica-se que, para todos os 14 picos de máxima absorbância do $\mathrm{Ho}_{2} \mathrm{O}_{3}$ analisados, $\left|\beta_{1}\right|$ ficou menor que $s \beta_{1} t_{\mathrm{st}}$, indicando que não houve tendência e demonstrando estabilidade por 9 meses, se mantido e armazenado à temperatura de $25^{\circ} \mathrm{C}$.

Verificou-se que o estudo de estabilidade a curto prazo foi mais crítico que a estabilidade a longo prazo, apresentando uma tendência nos resultados devido às diferenças de temperatura extremas.

\section{Caracterização do candidato a MRC}

Nesta etapa, foram distribuídas 3 ampolas do lote contendo a solução produzida para cada laboratório, portanto, 6 ampolas foram analisadas para obtenção do valor da caracterização.

Os resultados de caracterização dos 2 laboratórios foram analisados quanto à compatibilidade através do índice zeta, no qual, para 
Tabela 2. Valores de $\left|\beta_{l}\right|$ e $s \beta_{1} t_{\mathrm{st}}$ referentes à regressão linear da avaliação da estabilidade a curto prazo do lote candidato a MRC, para os 14 picos especificados na Figura 1

\begin{tabular}{|c|c|c|c|c|c|c|}
\hline \multirow{2}{*}{$\begin{array}{l}\text { Valor nominal } \\
\text { do pico }(\mathrm{nm})\end{array}$} & \multicolumn{2}{|c|}{$4^{\circ} \mathrm{C}$} & \multicolumn{2}{|c|}{$25^{\circ} \mathrm{C}$} & \multicolumn{2}{|c|}{$50^{\circ} \mathrm{C}$} \\
\hline & $\left|\beta_{l}\right| \times 10^{-5}$ & $\left(s \beta_{1} t_{\mathrm{st}}\right) \times 10^{-5}$ & $\left|\beta_{l}\right| \times 10^{-5}$ & $\left(s \beta_{1} t_{\mathrm{st}}\right) \times 10^{-5}$ & $\left|\beta_{1}\right| \times 10^{-5}$ & $\left(s \beta_{1} t_{\mathrm{st}}\right) \times 10^{-5}$ \\
\hline 241 & 18 & 34 & 12 & 30 & 29 & 38 \\
\hline 249 & 11 & 74 & 2 & 59 & 5 & 83 \\
\hline 278 & $47^{\mathrm{a}}$ & 37 & 8 & 29 & $61^{a}$ & 37 \\
\hline 287 & $33^{\mathrm{a}}$ & 32 & 28 & 30 & 29 & 37 \\
\hline 333 & 33 & 64 & 6 & 67 & 40 & 63 \\
\hline 345 & 51 & 102 & 80 & 112 & 39 & 108 \\
\hline 361 & 16 & 37 & $43^{a}$ & 36 & 24 & 51 \\
\hline 385 & 22 & 75 & 22 & 67 & 42 & 66 \\
\hline 416 & $54^{\mathrm{a}}$ & 44 & $47^{\mathrm{a}}$ & 45 & $69^{a}$ & 38 \\
\hline 451 & 23 & 99 & 7 & 105 & 3 & 116 \\
\hline 467 & 3 & 27 & 13 & 31 & 14 & 28 \\
\hline 485 & 9 & 31 & 27 & 33 & 26 & 33 \\
\hline 536 & $46^{\mathrm{a}}$ & 42 & 3 & 33 & 8 & 37 \\
\hline 640 & 22 & 61 & 8 & 45 & 7 & 50 \\
\hline
\end{tabular}

a Valores de $\left|\beta_{l}\right|$ maiores que $s \beta_{l} t_{s t}$.

Tabela 3. Valores $\left|\beta_{l}\right|$ e $s \beta_{l} t_{\text {st }}$ referentes à regressão linear da avaliação da estabilidade a longo prazo do lote candidato a MRC, para os 14 picos especificados na Figura 1

\begin{tabular}{ccc}
\hline $\begin{array}{c}\text { Valor nominal } \\
\text { do pico }(\mathrm{nm})\end{array}$ & $\left|\beta_{I}\right| \times 10^{-6}$ & $\left(s \beta_{1} t_{\mathrm{st}}\right) \times 10^{-6}$ \\
\hline 241 & 35 & 47 \\
249 & 32 & 82 \\
278 & 16 & 53 \\
287 & 22 & 68 \\
333 & 2 & 92 \\
345 & 8 & 119 \\
361 & 38 & 54 \\
385 & 77 & 88 \\
416 & 23 & 64 \\
451 & 25 & 150 \\
467 & 2 & 50 \\
485 & 4 & 55 \\
536 & 40 & 43 \\
640 & 4 & 64 \\
\hline
\end{tabular}

todos os picos, ficou confirmado que todos ficaram compatíveis. Dessa forma, os resultados dos laboratórios foram combinados por média ponderada, ${ }^{9}$ cujos valores constam na Tabela 4.

\section{Estimativa da incerteza do $\mathrm{MRC}$ de $\mathrm{Ho}_{2} \mathrm{O}_{3}$}

As incertezas padrão de cada estudo foram calculadas conforme ISO Guide $35 .{ }^{13}$ Para o estudo de estabilidade a longo prazo, a incerteza foi extrapolada em mais 3 meses, resultando num total de 12 meses. Dessa forma, o prazo de validade da solução de $\mathrm{Ho}_{2} \mathrm{O}_{3}$ foi estimado para 1 ano após a produção.

Os valores de cada incerteza padrão referentes aos estudos de certificação e a incerteza expandida do MRC estão apresentados na Tabela 5.

\section{Atribuição do valor de propriedade}

$\mathrm{O}$ valor de propriedade da solução de $\mathrm{Ho}_{2} \mathrm{O}_{3}$ produzida foi apresentado para cada um dos 14 picos de máxima absorbância,
Tabela 4. Resultados obtidos pelos laboratórios na etapa de caracterização e a média ponderada (dados em $\mathrm{nm}$ )

\begin{tabular}{ccc}
\hline $\begin{array}{c}\text { Valores obtidos } \\
\text { pelo Laraf }\end{array}$ & $\begin{array}{c}\text { Valores obtidos } \\
\text { pela Visomes }\end{array}$ & $\begin{array}{c}\text { Média } \\
\text { ponderada }\end{array}$ \\
\hline 241,25 & 241,14 & 241,192 \\
250,00 & 249,90 & 249,947 \\
278,22 & 278,12 & 278,167 \\
287,33 & 287,23 & 287,277 \\
333,57 & 333,48 & 333,526 \\
345,47 & 345,40 & 345,439 \\
361,33 & 361,25 & 361,289 \\
385,71 & 385,63 & 385,667 \\
416,35 & 416,26 & 416,304 \\
451,42 & 451,40 & 451,411 \\
467,88 & 467,82 & 467,848 \\
485,30 & 485,22 & 485,256 \\
536,62 & 536,55 & 536,582 \\
640,55 & 640,49 & 640,517 \\
\hline
\end{tabular}

nos comprimentos de onda certificados, sendo a média ponderada dos valores obtidos pelos 2 laboratórios. A incerteza da solução a ser considerada foi estabelecida como a maior incerteza encontrada dentre os 14 picos certificados: apenas um valor de incerteza expandida foi apresentado para os 14 picos. Os valores de propriedade dos 14 picos de máxima absorbância da solução de $\mathrm{Ho}_{2} \mathrm{O}_{3}$ produzida são apresentados na Tabela 6 .

\section{CONCLUSÃO}

Uma metodologia para preparo e envase em ampolas de vidro do material foi desenvolvida, e realizada a avaliação da homogeneidade e da estabilidade a curto e longo prazo, na qual a solução de $\mathrm{Ho}_{2} \mathrm{O}_{3}$ produzida demonstrou-se homogênea e estável por 9 meses. Com a avaliação da estabilidade a curto e a longo prazos foi demonstrado que o material se manteve estável durante o transporte por até 5 semanas em temperaturas elevadas, atingindo $50{ }^{\circ} \mathrm{C}$ e temperaturas mais baixas de até $4^{\circ} \mathrm{C}$, além de poder ser armazenado à temperatura ambiente por até um ano. $\mathrm{O}$ material produzido foi caracterizado por 
Tabela 5. Valores de incerteza-padrão dos 14 picos da solução produzida de $\mathrm{Ho}_{2} \mathrm{O}_{3}$ obtidos nos estudos de certificação e a incerteza expandida do MRC (dados em nm)

\begin{tabular}{cccccc}
\hline $\begin{array}{c}\text { Valor } \\
\text { nominal } \\
\text { do pico }\end{array}$ & $u_{\text {hom }}$ & $u_{\mathrm{ec}}$ & $u_{\mathrm{el}}$ & $u_{\text {car }}$ & $U_{\mathrm{MRC}}$ \\
\hline 241 & 0,004 & 0,006 & 0,008 & 0,044 & 0,09 \\
249 & 0,008 & 0,014 & 0,015 & 0,044 & 0,10 \\
278 & 0,003 & 0,021 & 0,010 & 0,044 & 0,10 \\
287 & 0,002 & 0,012 & 0,012 & 0,044 & 0,10 \\
333 & 0,004 & 0,011 & 0,017 & 0,047 & 0,10 \\
345 & 0,006 & 0,019 & 0,022 & 0,048 & 0,11 \\
361 & 0,002 & 0,015 & 0,010 & 0,045 & 0,10 \\
385 & 0,004 & 0,013 & 0,016 & 0,044 & 0,10 \\
416 & 0,006 & 0,024 & 0,012 & 0,046 & 0,11 \\
451 & 0,009 & 0,020 & 0,027 & 0,048 & 0,12 \\
467 & 0,003 & 0,005 & 0,009 & 0,044 & 0,09 \\
485 & 0,001 & 0,006 & 0,010 & 0,044 & 0,09 \\
536 & 0,003 & 0,016 & 0,008 & 0,044 & 0,10 \\
640 & 0,003 & 0,010 & 0,012 & 0,044 & 0,09 \\
\hline
\end{tabular}

Tabela 6. Valores de propriedade da solução de $\mathrm{Ho}_{2} \mathrm{O}_{3}$ produzida (dados em nm)

\begin{tabular}{cc}
\hline Valor de Propriedade & $U$ \\
\hline 241,19 & 0,12 \\
249,95 & 0,12 \\
278,17 & 0,12 \\
287,28 & 0,12 \\
333,53 & 0,12 \\
345,44 & 0,12 \\
361,29 & 0,12 \\
385,67 & 0,12 \\
416,30 & 0,12 \\
451,41 & 0,12 \\
467,85 & 0,12 \\
485,26 & 0,12 \\
536,58 & 0,12 \\
640,52 & 0,12 \\
\hline
\end{tabular}

meio de medições utilizando métodos independentes realizados em 2 laboratórios, de acordo com a Norma ABNT NBR ISO 17034, sendo obtidos os valores de propriedade dos 14 picos de máxima absorbância da solução de $\mathrm{Ho}_{2} \mathrm{O}_{3}$. A partir da combinação das incertezas padrão referentes à homogeneidade, estabilidade e da caracterização, a incerteza expandida do MRC foi estimada em $0,12 \mathrm{~nm}$, para um fator de abrangência $(k)$ igual a 2,00, em uma probabilidade de abrangência de aproximadamente $95 \%$.

\section{AGRADECIMENTOS}

APDA e TOA agradecem ao Conselho Nacional de Desenvolvimento Científico e Tecnológico - CNPq.

\section{REFERÊNCIAS}

1. Augusto, C. R.; Monteiro, T. M.; Rodrigues, J. M.; Cunha, V. S.; Congresso Interno do Inmetro, Rio de Janeiro, Brasil, 2010.

2. Gonçalves, D. E. F.; Dissertação de Mestrado, Instituto Nacional de Metrologia, Qualidade e Tecnologia, Brasil, 2018.

3. Travis, J. C.; Acosta, J. C.; Andor, G.; Bastie, J.; Blattner, P.; Chunnilall, C. J.; Crosson, S. C.; Duewer, D. L.; Early, E. A.; Hengstberger, F.; Kim, C. S.; Liedquist, L.; Manoocheri, F.; Mercader, F.; Monard, L. A. G.; Nevas, S.; Mito, A.; Nilsson, M.; Noël, M.; Rodriguez, A. C.; Ruíz, A.; Schirmacher, A.; Smith, M. V.; Valencia, G.; van Tonder, N.; Zwinkels, J.; J. Phys. Chem. Ref. Data 2005, 34, 41.

4. International Accreditation New Zealand; Technical Guide, Nova Zelândia, 2005.

5. European Pharmacopoeia; Ph.Eur., $9^{\text {th }}$ ed., França, 2017.

6. The United States Pharmacopoeia; National Formulary 35, 40, EUA, 2017

7. Weidner, V. R.; Mavrodineanu, R.; Mielenz, K. D.; Velapoldi, R. A.; Eckerle, K. L.; Adams, B.; NBS Special Publications 1986, 260-102, 69.

8. Weidner, V. R.; Mavrodineanu, R.; Mielenz, K. D.; Velapoldi, R. A.; Eckerle, K. L.; Adams, B.; J. Res. Natl. Bur. Stand. 1985, 90, 115.

9. Miller, J. N.; Miller, J. C.; Statistics and Chemometrics for Analytical Chemistry, $5^{\text {a }}$ ed., Person: Inglaterra, 2005.

10. ABNT NBR ISO 17034; Requisitos gerais para a competência de produtores de material de referência, $1^{\text {a }}$ ed., Rio de Janeiro, 2017.

11. ISO 13528; Statistical methods for use in proficiency testing by interlaboratory comparison, $2^{\mathrm{a}}$ ed., França, 2015.

12. Cox M. G.; Advanced Mathematical and Computational Tools in Metrology IV 2000, 53, 45.

13. ISO Guide 35; Reference materials - Guidance for characterization and assessment of homogeneity and stability; $4^{\mathrm{a}} \mathrm{ed}$., França, 2017. 